\title{
Dynamic Stability of Open-Loop Hopping
}

Jorge G. Cham ${ }^{1}$

e-mail: jgcham@robotics.caltech.edu

\section{Mark R. Cutkosky}

Center for Design Research, Stanford University,

Stanford, CA 94305-2232
Simulations and physical robots have shown that hopping and running are possible without sensory feedback. However, stable behavior is often limited to a certain range of the parameters of the open-loop system. Even the simplest of hopping systems can exhibit unstable behavior that results in unpredictable nonperiodic motion as system parameters are adjusted. This paper analyzes the stability of a simplified vertical hopping model driven by an open-loop, feedforward motor pattern. Periodic orbits of the resulting hybrid system are analyzed through a generalized formula for the system's Poincare Map and Jacobian. The observed behavior is validated experimentally in a physical pneumatically actuated hopping machine. This approach leads to observations on the stability of this and similar systems, revealing inherent limitations of open-loop hopping and providing insights that can inform the design and control of dynamic legged robots capable of rapid and robust locomotion. [DOI: 10.1115/1.2718237]

\section{Introduction}

Animals display remarkable agility and versatility when traversing unstructured environments. This locomotion, despite its highly dynamic nature, is not always achieved through highbandwidth closed-loop control. Studies of animal locomotion have shown that neural circuits called Central Pattern Generators, which can generate motor activation patterns in the absence of sensory feedback, play a large role in creating and regulating rhythmic tasks such as walking and running [1-4]. In fact, robot prototypes such as the Sprawl robots [5] (see Fig. 1) and others $[6,7]$ have shown that fast robust locomotion is possible entirely without feedback. The Sprawl robots are capable of speeds of over five body-lengths/s while being driven by an open-loop motor pattern [5]. The key is in the proper design of the mechanical system, relying on limb arrangement and passive viscoelastic properties, mechanisms known as "preflexes," to reject disturbances [8-10].

Although open-loop locomotion is possible, stable behavior is often limited to a certain range of the system parameters. Doubleperiod oscillations and unstable behavior that leads to crashing have been observed in the Sprawl robots for certain values of the stride frequency and leg configuration. Thus, there is a need to understand the mechanics in dynamic locomotion that determine the boundaries for stable motion under open-loop control.

In this paper, we analyze the stability of a simplified vertical hopping model that is driven by an open-loop motor pattern. Analyzing simplified models of locomotion that capture the underlying dynamics can lead to insights that explain the observed behavior of more complex systems. This approach is supported by studies that show that running animals of different size, morphology, and number of legs all produce ground reaction forces that can be characterized by the dynamics of a spring-loaded inverted pendulum (SLIP) model [11]. The SLIP is a simple model that consists of a point mass with a compliant massless leg, akin to a pogo stick. Moreover, Raibert [12] showed that it is possible to decouple stabilization in the vertical and horizontal directions for a SLIP-like hopping robot. This motivates the study of the vertical hopping model as a starting point for understanding the basic dynamics of running.

${ }^{1}$ Currently with the Department of Mechanical Engineering, California Institute of Technology, Pasadena, CA

Contributed by the Dynamic Systems, Measurement, and Control Division of ASME for publication in the Journal of Dynamic Systems, Measurement, and ConTROL. Manuscript received September 8, 2004; final manuscript received September 21, 2006. Associate Editor Jordan Berg.
The vertical hopping model, though simple in appearance, exhibits a rich set of dynamics due to the leg's intermittent contact with the ground, resulting in a hybrid dynamical system [13]. For the open-loop hopper considered in this study, the system undergoes changes in its equations of motion due not only to physical events (coming into and out of contact with the ground) but also due to time-based events (activation and deactivation of the openloop forcing function). In the following sections, we review previous work and present the vertical hopping model analyzed. Periodic orbits of the hybrid system and their stability are then characterized using a generalized form of the system's Poincare Map (also called a Return Map) and its Jacobian. These observations are then validated in a pneumatically actuated hopping machine. Finally, we present conclusions and future work.

By providing insights into the conditions for stability of this and similar systems and revealing inherent limitations of openloop hopping, this analysis can help inform the study of dynamic locomotion and the design and control of future legged robots.

\section{Related Work}

Raibert's pioneering development of one-legged robots [12] that could achieve stable hopping using only a simple set of control laws sparked much interest in understanding the nature of hopping dynamics. The robot consists of a body, an actuated hip, and a double-acting pneumatic cylinder for a leg that acts as an air spring when the robot first contacts the ground. When the spring is sensed to be at maximum compression, the robot applies pressure to the cylinder and pushes against the ground. Raibert found that vertical hopping height was a function of how long the cylinder was pressurized.

Koditschek and Buehler [14] showed that a simplified dynamic model of Raibert's hopper, in which thrust by a nonlinear spring was initiated through closed-loop sensory feedback, belonged to a special class of dynamical system with ensured stability. Vakakis et al. [15] showed that chaotic, or non-period-1 behavior can occur for certain sets of the parameters of a similar model.

Ringrose [16] showed through simulation and a prototype robot that a vertical hopper can maintain stable hopping without sensory feedback. Using a simplified model in which thrust is applied through a periodic impulsive change in leg length, Ringrose analyzed the stability of the hopping height and phase (motion of the hopper relative to the cycle of the open-loop motor pattern) separately, in both cases applying simplifications that assume low damping. The analysis concluded that the hopper is unstable if 


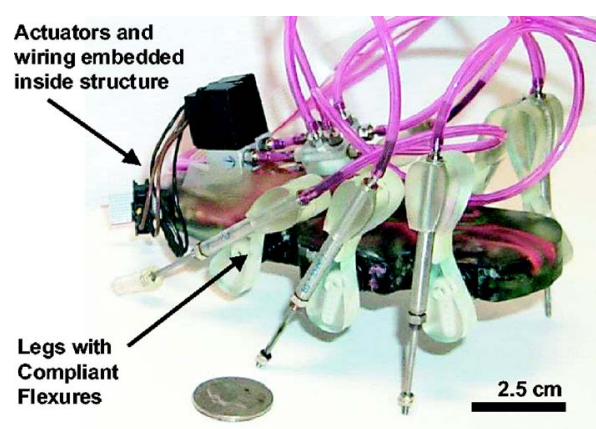

Fig. 1 Sprawl robots, which achieve fast and robust locomotion without sensory feedback

thrust initiation occurs at maximum compression, and assumes that trajectories in which thrust starts after maximum compression do not exist.

Berkemeier and Desai [17] analyze a similar model and concluded that the open-loop hopper reaches maximum hopping height when thrust is applied at maximum spring compression. Using perturbation methods that assume low values of damping, they also found that the motion is stable, without feedback, when the force is applied prior to the maximum compression of the spring and unstable when the force is applied after maximum compression.

Komsuoglu and Koditschek [18] analyze the stability of a openloop vertical hopper in which thrust is effected by clock-driven changes in the leg spring's stiffness. Neglecting gravity for simplification, they find conditions for stability, which include the necessary presence of viscous friction.

Despite this history of analysis, several questions remain: How does performance vary as a function of the open-loop motor pattern? What are the trade-offs in stability? What is the effect of damping and gravity in the analysis? What are insights that can lead to design and control strategies for legged robots?

\section{Simplified Model for Open-Loop Hopping}

In running insects, and in the Sprawl family of robots, viscoelastic materials dissipate substantial amounts of energy per cycle, contributing to stability $[19,20]$. Typical dimensionless damping ratios for insects are on the order of 0.3 [21]. The model studied here includes significant damping (damping ratio of 0.2 ), and gravity (of similar magnitude as the thrust force). Both of these assumptions make finding closed forms for the steady-state solutions intractable, though it is still possible to find analytical expressions for the multivariable Jacobian of the Poincare Map. In addition, this analysis considers the case where thrust is deactivated before take-off, which was not considered by previous analyses.

3.1 Model Description. The simplified vertical hopping model used in this analysis is illustrated in Fig. 2. As shown, the model consists of a point mass, $m$, subject to gravitational acceleration, $g$, and attached to a massless leg which contains in parallel a damping element, $b$, a linear spring $k$, and a force source, $s(t)$ (it is assumed that the mass of the leg is neglible compared to the mass of the body). The leg has a nominal rest length of $r_{0}$. The leg comes into contact with the ground (landing) when $h=0$ (the ground is placed at $h=-r_{0}$ ). Take-off is assumed to occur when the leg length exceeds the nominal rest length. With this assumption, the presence of damping in the leg model causes the ground reaction force to be negative in the moments before take-off. This effect was not found to be significant in the trajectories studied.

The forcing source $s(t)$ is a square wave signal with period $\tau$

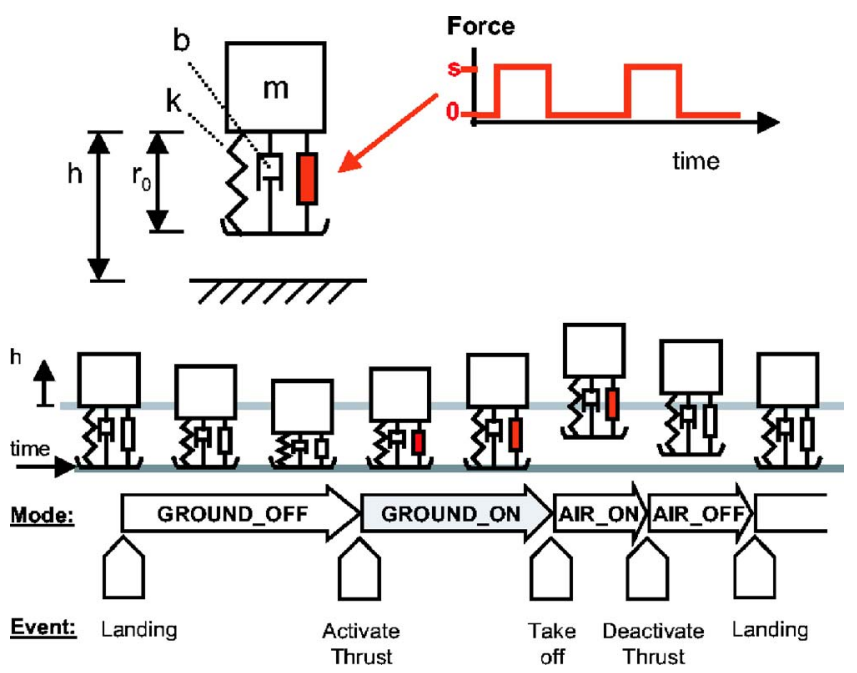

Fig. 2 The vertical hopping model used for analysis

and thrust duration $t_{\mathrm{on}}$, as shown in Fig. 2. The differential equations that govern this system can be written, in normalized coordinates $y=h / g$ and $f(t)=s(t) /(m g)$, as

$$
\dot{Y}=A Y+B
$$

where $Y$ is the normalized height and vertical velocity of the point mass, $[y \dot{y}]^{T}$, and $A$ and $B$ are defined as

$$
A=\left[\begin{array}{cc}
0 & 1 \\
-w^{2} & -2 \zeta w
\end{array}\right] \quad B=\left[\begin{array}{c}
0 \\
f(t)-1
\end{array}\right] \quad(y \leqslant 0)
$$

during the stance, or ground-contact phase, and

$$
A=\left[\begin{array}{ll}
0 & 1 \\
0 & 0
\end{array}\right] \quad B=\left[\begin{array}{c}
0 \\
-1
\end{array}\right] \quad(y>0)
$$

during the airborne, or ballistic, phase, $w$ is the natural frequency and $\zeta$ is the damping ratio of the mass-spring-damper system. The thrust force $f(t)$ is determined by the open-loop motor control pattern

$$
f(t)= \begin{cases}T_{n} & 0<t \leqslant t_{\text {on }} \\ 0 & \text { otherwise }\end{cases}
$$

where $T_{n}$ is the normalized thrust magnitude. Here, $t$ is reset to $t=0$ when $t$ reaches $\tau$. This system is treated as a piecewise affine linear hybrid dynamic system with continuity of state at the mode transitions [13]. We define three modes of the system, since $f(t)$ has no effect while the hopper is in the air: "AIR" (airborne phase), "GND_ON" (stance phase with active thrust), and " GND_OFF" (stance phase with zero thrust).

Since changes in the equation of motion are given by conditions in both $Y$ and conditions in $t$, we define the state space of our system, $X$, to include a proxy variable to represent time, that is added to the previous variable $Y$

$$
X=\left[\begin{array}{l}
t \\
Y
\end{array}\right]
$$

The equations of motion for the system can thus be written as

$$
\dot{X}=\left[\begin{array}{ll}
0 & 0 \\
0 & A
\end{array}\right] X+\left[\begin{array}{l}
1 \\
B
\end{array}\right]
$$

If $A$ is invertible (as is the case during the stance phases), the time solution for this differential equation is given by 


$$
X(t)=\left[\begin{array}{cc}
1 & 0 \\
0 & e^{A t}
\end{array}\right]\left(X_{0}-X_{e}\right)+X_{e}+\left[\begin{array}{l}
t \\
0
\end{array}\right]
$$

where $e^{A t}$ is the matrix exponential, $X_{0}$ is the initial state, and $X_{e}$ is the equilibrium state of the system, given by

$$
X_{e}=\left[\begin{array}{c}
0 \\
-A^{-1} B
\end{array}\right]
$$

Note that the number zero in the expressions can represent both the scalar number zero and the zero matrix as needed to maintain consistency. The number " 1 " always represents the scalar number one.

During the AIR phase, the solution to the system is given by

$$
X(t)=\left[\begin{array}{lll}
1 & 0 & 0 \\
0 & 1 & t \\
0 & 0 & 1
\end{array}\right] X_{0}-\left[\begin{array}{c}
-t \\
t^{2} / 2 \\
t
\end{array}\right]
$$

Inclusion of time in the state space of the system will allow us to define the Poincare Map anywhere along the trajectory, as shown in the following section. Previous studies of the open-loop hopper limited the formulation of the Poincare Map to the moment of thrust activation.

3.2 Derivation of Poincare Map for Periodic Orbits. We seek to find periodic orbits of the above-mentioned hybrid system and to analyze their stability with respect to the parameters of the open-loop motor pattern. Steady-state orbits are found using the Poincare method by defining a Poincare Map

$$
X_{n+1}=F\left(X_{n}\right)
$$

which maps the state of the system between subsequent intersections of the trajectory with a Poincare Section [22]. The Poincare Section is commonly defined as an (n-1)-dimensional manifold in $(n)$-dimensional space that is approximately orthogonal, or "transversal," to the trajectory at the point of intersection. The function $F$ is found by the forward integration of the equations of motion, with the assumption that both the start and end condition lie on the Poincare Section. Steady-state orbits are represented by the points that satisfy the condition

$$
X^{*}=F\left(X^{*}\right)
$$

Since a steady-state trajectory of the above-mentioned system can undergo an indeterminate number of mode transitions between intersections with the Poincare Section, finding a general analytical expression for $F$ is not possible. The approach taken is to first enumerate the sequences of mode transitions that are of interest to our analysis [18]. We then study the steady-state trajectories within each sequence, assuming perturbations small enough that do not cause the sequence to change. In particular, we focus on two possible sequences that represent "normal" hopping, which we denote as "Long Thrust" and "Short Thrust." In Long Thrust, the hopper first lands, then activates thrust during stance, and the thrust duration is long enough to continue until or past take-off, such that the mode sequence is given by

$$
\text { Long Thrust: } \quad \text { AIR, GND _ OFF, GND _ ON }\}
$$

In Short Thrust, the hopper deactivates thrust before take-off, such that there is an additional GND_OFF mode in the sequence

$$
\text { Short Thrust: } \quad \text { AIR,GND_OFF,GND_ON,GND_OFF }
$$

To find $F$ for both sequences, we first define variables for the state of the system at the mode transitions

$$
X_{\text {landing }} X_{\text {takeoff }} X_{\text {activate }} X_{\text {deactivate }}
$$

where $X_{\text {landing }}$ is the state at the instant the hopper contacts the ground, etc., as illustrated by Fig. 3. To derive the Poincare Maps, we find the individual expressions that map the state from one

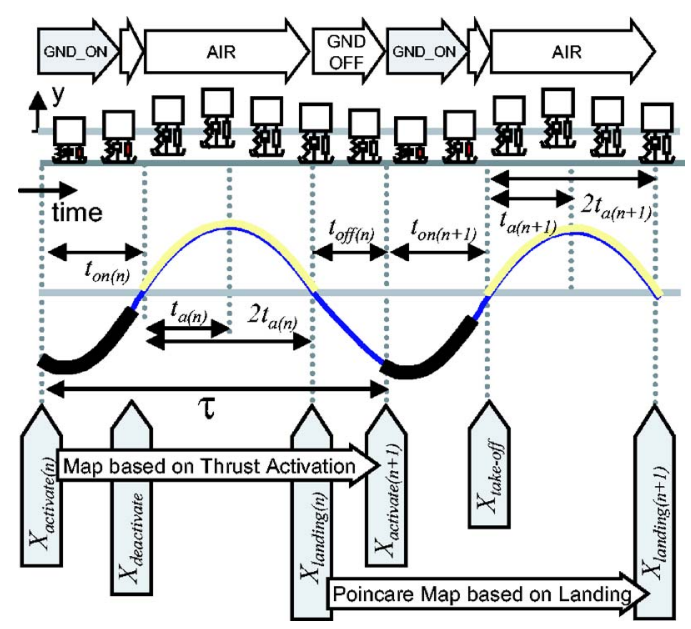

Fig. 3 Sample time history of the vertical hopper

mode transition to the next, using Eqs. (7) and (9), depending on the mode. We then concatenate these expressions in the appropriate sequence to find the Poincare Map.

In this case, we establish the Poincare Section at the moment that the hopper takes off. This will allow us to simplify the search for steady-state orbits. $F$ will map $X_{\text {takeoff }(n)}$, the state at takeoff in the $n$th cycle, to, $X_{\text {takeoff }(n+1)}$ the state at the following takeoff. We introduce the variables $\Delta t_{\text {on }}, \Delta t_{\text {off }}$, and $\Delta t_{\text {off } 2}$ to represent the time duration of the active and inactive stance modes, and $\Delta t_{a}$ as one half of the time duration of the airborne phase. For the Long Thrust case, $F$ is found to be

$$
\begin{aligned}
X_{\text {takeoff }(n+1)} & {\left[\begin{array}{cc}
1 & 0 \\
0 & e^{A\left(\Delta t_{\text {on }}+\Delta t_{\text {off }}\right)}
\end{array}\right]\left(\left[\begin{array}{ccc}
1 & 0 & 0 \\
0 & 1 & 2 \Delta t_{a} \\
0 & 0 & 1
\end{array}\right] X_{\text {takeoff }(n)}\right.} \\
& \left.-\left[\begin{array}{c}
-2 \Delta t_{a} \\
2\left(\Delta t_{a}\right)^{2} \\
2 \Delta t_{a}
\end{array}\right]-X_{\text {eoff }}\right)+\left[\begin{array}{cc}
1 & 0 \\
0 & e^{A \Delta t_{\text {on }}}
\end{array}\right]\left(X_{\text {eoff }}-X_{\text {eon }}\right) \\
& +X_{\text {eon }}+\left[\begin{array}{c}
\Delta t_{\text {on }}+\Delta t_{\text {off }} \\
0
\end{array}\right]
\end{aligned}
$$

where $X_{\text {eoff }}$ and $X_{\text {eon }}$ are the equilibrium states of the stance phases, according to Eq. (8). To reduce the number of equations, we impose the steady-state constraint that, at takeoff,

$$
X_{\text {takeoff }}=\left[\begin{array}{c}
t_{\text {takeoff }} \\
0 \\
\Delta t_{a}
\end{array}\right]
$$

and that the total cycle duration, or stride period, is $\tau$

$$
t_{\text {takeoff }(n-1)}=t_{\text {takeoff }(n)}+\tau
$$

such that the first row of Eq. (15) becomes

$$
\Delta t_{\text {on }}+\Delta t_{\text {off }}+2 \Delta t_{a}=\tau
$$

which we can substitute into the second and third rows of Eq. (15) to obtain

$$
\begin{aligned}
{\left[\begin{array}{c}
0 \\
\Delta t_{0}
\end{array}\right]=} & -e^{A\left(\tau-2 \Delta t_{a}\right)}\left(\left[\begin{array}{c}
0 \\
\Delta t_{a}
\end{array}\right]+Y_{\text {eoff }}\right)+e^{A\left(\tau-2 \Delta t_{a}-\Delta t_{\mathrm{off}}\right)}\left(Y_{\text {eoff }}-Y_{\text {eon }}\right) \\
& +Y_{\text {eon }}
\end{aligned}
$$

where $Y_{\text {eoff }}$ and $Y_{\text {eon }}$ are $X_{\text {eoff }}$ and $X_{\text {eon }}$ with the first row removed. With this equation, we can now numerically solve for a solution vector $\left[\Delta t_{\text {off }}, \Delta t_{a}\right]^{T}$ to find the steady-state trajectory for a given 
value of the stride period.

For the Short Thrust case, the Poincare Map based on the instant of takeoff can be similarly found and manipulated to obtain the following set of equations

$$
\begin{aligned}
{\left[\begin{array}{c}
0 \\
\Delta t_{a}
\end{array}\right]=} & -e^{A\left(\tau-2 \Delta t_{a}\right)}\left(\left[\begin{array}{c}
0 \\
\Delta t_{a}
\end{array}\right]+Y_{\text {eoff }}\right)+e^{A\left(\tau-2 \Delta t_{a}-\Delta t_{\mathrm{off}}\right)}\left(Y_{\mathrm{eoff}}-Y_{\mathrm{eon}}\right) \\
& +e^{A\left(\tau-2 \Delta t_{a}-\Delta t_{\mathrm{off}}-\Delta t_{\mathrm{on}}\right)}\left(Y_{\text {eon }}-Y_{\text {eoff }}\right)+Y_{\text {eoff }}
\end{aligned}
$$

which can be numerically solved for the same solution vector $\left[\Delta t_{\text {off }} \Delta t_{a}\right]^{T}$, since $\Delta t_{\text {on }}$ is a known system parameter.

3.3 Derivation of Jacobians for Local Stability. Solutions found using the above equations represent steady-state trajectories, or periodic orbits, of the system that follow the prescribed mode sequences. Small disturbances, however, can cause the system to either deviate away from or converge back to the steadystate trajectory. This local stability can be determined using Floquet Analysis [22], which uses the multivariable derivative, or Jacobian, of the Poincare Map, given by

$$
J=\frac{\partial X_{n+1}}{\partial X_{n}}=\frac{\partial F}{\partial X^{n}}
$$

This Jacobian, in essence, maps small disturbances from the steady-state trajectory at the Poincare Section from one cycle to the next. Local stability is given by the eigenvalues, also called Floquet multipliers, of $J$. The steady-state trajectory is said to be asymptotically stable if $\left|\lambda_{i}\right|<1$ and unstable if at least one eigenvalue $\left|\lambda_{i}\right|>1$.

A useful method to determine the Jacobian is to apply the chain rule to write the Jacobian as the product of individual "sensitivity" matrices [23]

$$
J\left(X^{*}\right)=J_{m}\left(X^{*}\right) J_{m-1}\left(X^{*}\right) \cdots J_{1}\left(X^{*}\right)
$$

where $m$ is the number of modes along the steady-state trajectory. Similar to the Jacobian for the overall Poincare Map, each individual sensitivity matrix is the multivariable derivative of the map between two mode transitions

$$
J_{i}\left(x^{*}\right)=\left.\frac{\partial M_{i}}{\partial X_{i}}\right|_{X^{*}}=\left.\frac{\partial X_{i+1}}{\partial X_{i}}\right|_{x^{*}} i=1,2, \ldots, m
$$

where $M_{i}$ is the map between the states $X_{i}$ and $X_{i+1}$ at mode transitions $(i)$ and $(i+1)$, respectively

$$
M_{i}: \quad X_{i}\left(t_{i}\right) \rightarrow X_{i+1}\left(t_{i+1}\right)
$$

The Jacobian of the Poincare Map is invariable in terms of its eigenvalues to the choice of Poincare Map [23]. This allows us to derive analytically the individual sensitivity matrices and arrange them in the order that yields the most useful analytical expression for each of the two mode sequences.

We now derive a general formula that can be used to obtain the sensitivity matrix between two arbitrary mode transitions along a nominal trajectory for linear, time-invariant systems given by Eq. (6), where $A$ is invertible. As illustrated in Fig. 4, the mode transitions are assumed to be linear conditions given by the intersection of the trajectory with planes in the state space defined by the unit vector $C$ and the scalar constant $c_{0}$

$$
C_{i}^{T} X_{i}=c_{0(i)}
$$

(e.g., the condition for landing is that $y=0$, so that $C_{i}^{T}=\left[\begin{array}{lll}0 & 1 & 0\end{array}\right]^{T}$ and $\left.c_{0(i)}=0\right)$. This plane is assumed to be approximately orthogonal to the steady-state trajectory at the mode transitions, such that

$$
C_{i}^{T} \dot{X}_{i} \neq 0
$$

Since the time at the end of the mode is given by

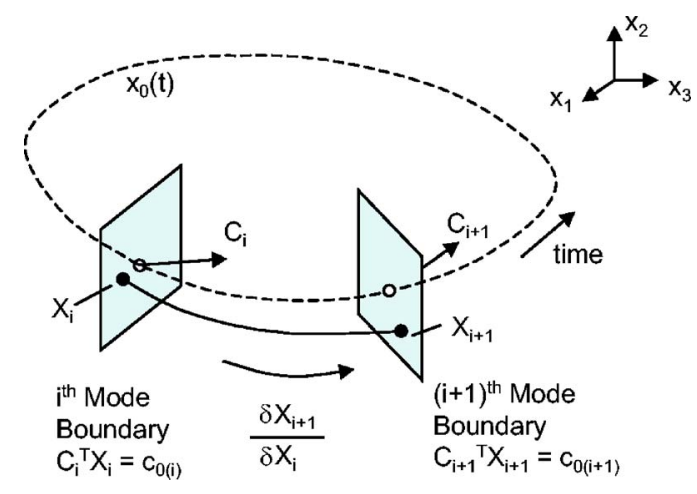

Fig. 4 Illustration of Jacobian of map between two mode boundaries

$$
t_{i+1}=t_{i}+\Delta_{t}
$$

where $\Delta$, is the scalar time duration of the trajectory between the two mode boundaries, the map between two mode boundaries is given by the time solution of the system

$$
X_{i+1}=\left[\begin{array}{cc}
1 & 0 \\
0 & e^{A \Delta_{t}}
\end{array}\right]\left(X_{i}-X_{e}\right)+X_{e}+\left[\begin{array}{c}
\Delta_{t} \\
0
\end{array}\right]
$$

To find the Jacobian of this map, we take the derivative with respect to the initial state, $X_{i}$, taking advantage of the derivative properties of the matrix exponential

$$
\frac{\partial e^{A f(s)}}{\partial s}=A e^{A f(s)} \frac{\partial f}{\partial s}=e^{A f(s)} A \frac{\partial f}{\partial s}
$$

resulting in

$$
\frac{\partial X_{i+1}}{\partial X_{i}}=\left[\begin{array}{cc}
0 & 0 \\
0 & A e^{A \Delta_{t}}
\end{array}\right]\left(X_{i}-X_{e}\right) \frac{\partial \Delta_{t}}{\partial X_{i}}+\left[\begin{array}{cc}
1 & 0 \\
0 & e^{A \Delta_{t}}
\end{array}\right]+\left[\begin{array}{l}
1 \\
0
\end{array}\right] \frac{\partial \Delta_{t}}{\partial X_{i}}
$$

This expression can be rewritten in a much simpler form as

$$
\frac{\partial X_{i+1}}{\partial X_{i}}=\dot{X}_{i+1} \frac{\partial \Delta_{t}}{\partial X_{i}}+\left[\begin{array}{cc}
1 & 0 \\
0 & e^{A \Delta_{t}}
\end{array}\right]
$$

where $\dot{X}_{i+1}$ is the rate of change of the state at the second mode boundary. To show this, we start by grouping together the terms in Eq. (30) with $\partial \Delta_{t} / \partial X_{i}$, resulting in

$$
\frac{\partial X_{i+1}}{\partial X_{i}}=\left(\left[\begin{array}{cc}
0 & 0 \\
0 & A e^{A \Delta_{t}}
\end{array}\right]\left(X_{i}-X_{e}\right)+\left[\begin{array}{l}
1 \\
0
\end{array}\right]\right) \frac{\partial \Delta_{t}}{\partial X_{i}}+\left[\begin{array}{cc}
1 & 0 \\
0 & e^{A \Delta_{t}}
\end{array}\right]
$$

The first product of the first term can be rewritten as

$$
\left[\begin{array}{cc}
0 & 0 \\
0 & A e^{A \Delta_{t}}
\end{array}\right]\left(X_{t}-X_{e}\right)+\left[\begin{array}{l}
1 \\
0
\end{array}\right]=\left[\begin{array}{c}
0 \\
A e^{A \Delta_{t}}\left(Y_{i}-Y_{e}\right)
\end{array}\right]+\left[\begin{array}{l}
1 \\
0
\end{array}\right]
$$

where $Y_{i}$ is the physical state space (without the time variable included) at the first mode boundary. We can rewrite this term by noting that

$$
A e^{A \Delta_{t}}\left(Y_{i}-Y_{e}\right)=A\left(Y_{i+1}-Y_{e}\right)=\dot{Y}_{i+1}
$$

resulting in

$$
\left[\begin{array}{c}
0 \\
A e^{A \Delta_{t}\left(Y_{i}-Y_{e}\right)}
\end{array}\right]+\left[\begin{array}{c}
1 \\
0
\end{array}\right]=\left[\begin{array}{c}
0 \\
\dot{Y}_{i+1}
\end{array}\right]+\left[\begin{array}{c}
1 \\
0
\end{array}\right]=\left[\begin{array}{c}
1 \\
\dot{Y}_{i+1}
\end{array}\right]=\dot{X}_{i+1}
$$

and 


$$
\begin{aligned}
\frac{\partial X_{i+1}}{\partial X_{i}} & =\left(\left[\begin{array}{cc}
0 & 0 \\
0 & A e^{A \Delta_{t}}
\end{array}\right]\left(X_{n}-X_{e}\right)+\left[\begin{array}{l}
1 \\
0
\end{array}\right]\right) \frac{\partial \Delta_{t}}{\partial X_{n}}+\left[\begin{array}{cc}
1 & 0 \\
0 & e^{A \Delta_{t}}
\end{array}\right] \\
& =\dot{X}_{i+1} \frac{\partial \Delta_{t}}{\partial X_{t}}+\left[\begin{array}{cc}
1 & 0 \\
0 & e^{A \Delta_{t}}
\end{array}\right]
\end{aligned}
$$

To find the term $\partial \Delta_{t} / \partial X_{i}$, we first make use of the constraint equation at the second boundary

$$
C_{i-1}^{T} X_{i+1}=c_{0(i+1)}
$$

and then, noting that taking the derivative with respect to $X_{n}$ results in

$$
\frac{\partial}{\partial X_{i}}\left(C_{i+1}^{T} X_{i+1}\right)=C_{i+1}^{T} \frac{\partial X_{i+1}}{\partial X_{i}}=\frac{\partial c_{0(i+1)}}{\partial X_{i}}=0
$$

we can premultiply Eq. (36) by $c_{i+1}^{T}$, to yield

$$
C_{i+1}^{T} \frac{\partial X_{i+1}}{\partial X_{i}}=C_{i+1}^{T}\left(\dot{X}_{i+1} \frac{\partial \Delta_{t}}{\partial X_{i}}+\left[\begin{array}{cc}
1 & 0 \\
0 & e^{A \Delta_{t}}
\end{array}\right]\right)=0
$$

This gives us a way to solve for $\left(\partial \Delta_{t}\right) /\left(\partial X_{i}\right)$

$$
\begin{gathered}
C_{i+1}^{T}\left(\dot{X}_{i+1} \frac{\partial \Delta_{t}}{\partial X_{i}}+\left[\begin{array}{cc}
1 & 0 \\
0 & e^{A \Delta_{t}}
\end{array}\right]\right)=0 \\
C_{i+1}^{T} \dot{X}_{i+1} \frac{\partial \Delta_{t}}{\partial X_{i}}+C_{i+1}^{T}\left[\begin{array}{cc}
1 & 0 \\
0 & e^{A \Delta_{t}}
\end{array}\right]=0 \\
\frac{\partial \Delta_{t}}{\partial X_{i}}=-\left(C_{i+1}^{T} \dot{X}_{i+1}\right)^{-1} C_{n+1}^{T}\left[\begin{array}{cc}
1 & 0 \\
0 & e^{A \Delta_{t}}
\end{array}\right]
\end{gathered}
$$

The condition for orthogonality ensures that $\left(C_{i+1}^{T} \dot{X}_{i+1}\right)^{-1}$ is finite. Thus, the Jacobian can be rewritten by substituting Eq. (42) into Eq. (36), resulting in

$$
\frac{\partial X_{i+1}}{\partial X_{i}}=-\dot{X}_{i+1}\left(C_{i+1}^{T} \dot{X}_{i+1}\right)^{-1} C_{i+1}^{T}\left[\begin{array}{cc}
1 & 0 \\
0 & e^{A \Delta_{t}}
\end{array}\right]+\left[\begin{array}{cc}
1 & 0 \\
0 & e^{A \Delta_{t}}
\end{array}\right]
$$

We can further simplify by factorization

$$
\frac{\partial X_{i+1}}{\partial X_{i}}=-\left(C_{i+1}^{T} \dot{X}_{i+1}\right)^{-1}\left(\dot{X}_{i+1} C_{i+1}^{T}-C_{i+1}^{T} \dot{X}_{i+1} I\right)\left[\begin{array}{cc}
1 & 0 \\
0 & e^{A \Delta_{t}}
\end{array}\right]
$$

where $I$ is the identity matrix. The above formulation ensures that the output space of this Jacobian lies in the plane described by $C_{i+1}$. Since any initial disturbance $\left(\partial X_{i}\right)$ must lie in the plane of the $i$ th mode transition, to ensure correctness the formula in Eq. (44) is postmultiplied by a projection matrix $P$ that projects the input space unto the plane given by $C_{i}$

$$
P=\left(I-C_{i} C_{i}^{T}\right)
$$

We can now use this general formula to derive expressions for the following individual sensitivity matrices

$$
\frac{\partial X_{\text {takeoff }}}{\partial X_{\text {deactivate }}} \frac{\partial X_{\text {takeoff }}}{\partial X_{\text {activate }}} \frac{\partial X_{\text {activate }}}{\partial X_{\text {landing }}} \frac{\partial X_{\text {deactivate }}}{\partial X_{\text {activate }}}
$$

For the AIR phase, where $A$ is not invertible, we follow a similar methodology to differentiate Eq. (9), resulting in

$$
\frac{\partial X_{\text {landing }}}{\partial X_{\text {takeoff }}}=\left[\begin{array}{ccc}
1 & 0 & 2 \\
0 & 0 & 0 \\
0 & 0 & -1
\end{array}\right]
$$

In our analysis, we will base the Poincare Map for the Long Thrust case on the instant of thrust activation to obtain the Jacobian $J_{L T}$

$$
J_{L T}=\frac{\partial X_{\text {activate }(n+1)}}{\partial X_{\text {activate }(n)}}=\frac{\partial X_{\text {activate }}}{\partial X_{\text {landing }}} \cdot \frac{\partial X_{\text {landing }}}{\partial X_{\text {takeoff }}} \cdot \frac{\partial X_{\text {takeoff }}}{\partial X_{\text {activate }}}
$$

Effect of period on "Long" Thrust

(f: $1.50 w: 30.0 \zeta: 0.20$ )
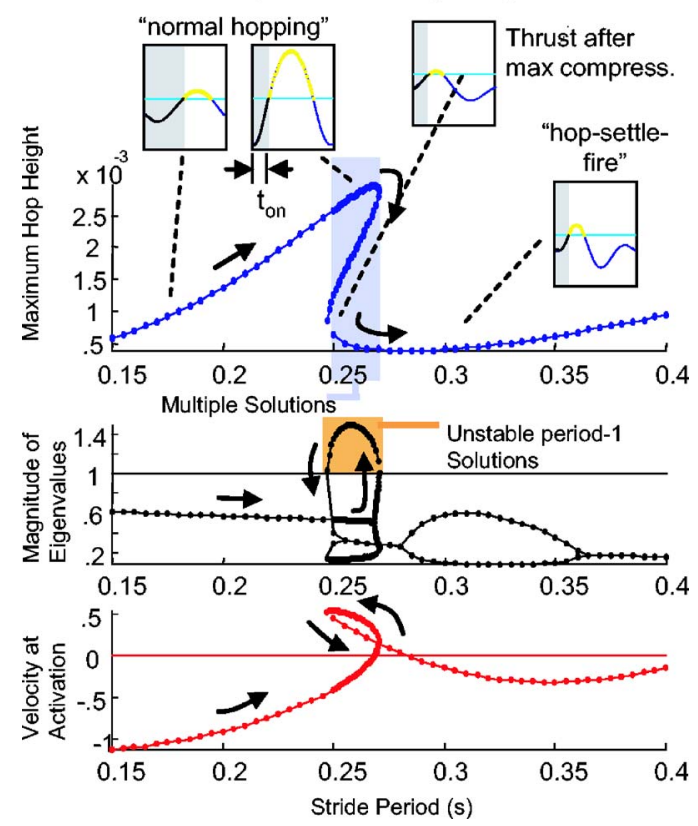

Fig. 5 Steady-state solutions as a function of stride period for the "Long Thrust" case

For the Short Thrust case, we will base the Poincare Map on the instant of landing, to obtain the Jacobian $J_{S T}$

$$
J_{S T}=\frac{\partial X_{\text {landing }(n+1)}}{\partial X_{\text {landing }(n)}}=\frac{\partial X_{\text {landing }}}{\partial X_{\text {takeoff }}} \cdot \frac{\partial X_{\text {takeoff }}}{\partial X_{\text {deactivate }}} \cdot \frac{\partial X_{\text {deactivate }}}{\partial X_{\text {activate }}} \cdot \frac{\partial X_{\text {activate }}}{\partial X_{\text {landing }}}
$$

As will be shown later, these choices for the location of the Poincare Map will result in analytical expressions from which approximate conclusions can be more easily derived. It is important to note that one of the eigenvalues of these Jacobian matrices will be 1 , which will correspond to the proxy variable for time added to the state space.

\section{Periodic Orbit Solutions}

4.1 Effects of Varying Stride Period. Using the above-found expressions for the Poincare Maps and Jacobian, steady-state solutions for a range of periods of the open-loop motor pattern were found numerically, given a set of prototypical system parameters, using the "fsolve" function in MATLAB" with a tolerance of $10^{-8}$.

For the Long Thrust case, Fig. 5 illustrates the effects of changing the open-loop stride period (in steady state, the stride period or orbit period is equal to the period set by the motor pattern). For short stride periods, hopping height starts out very small, as shown in the top plot. At these periods, thrust application starts well before maximum compression, given by the negative velocity at thrust application (in Fig. 5, this velocity is normalized by the magnitude of the takeoff velocity). These solutions are termed "Regular Hopping" as they represent a normal, desired mode of hopping behavior. As the stride period is increased, hopping height increases, and velocity at thrust activation approaches zero. Finally, at a certain period (near $275 \mathrm{~ms}$ period), height is maximized when velocity at thrust application is nearly zero (corresponding to thrust activation at maximum compression), as predicted by previous studies. However, as the period approaches $275 \mathrm{~ms}$ the magnitude of the eigenvalues quickly increases and the solutions become unstable. Simulations of the hopper, though, never reach this point. As shown in the figure, other solutions to the state-steady conditions become available at a period near 


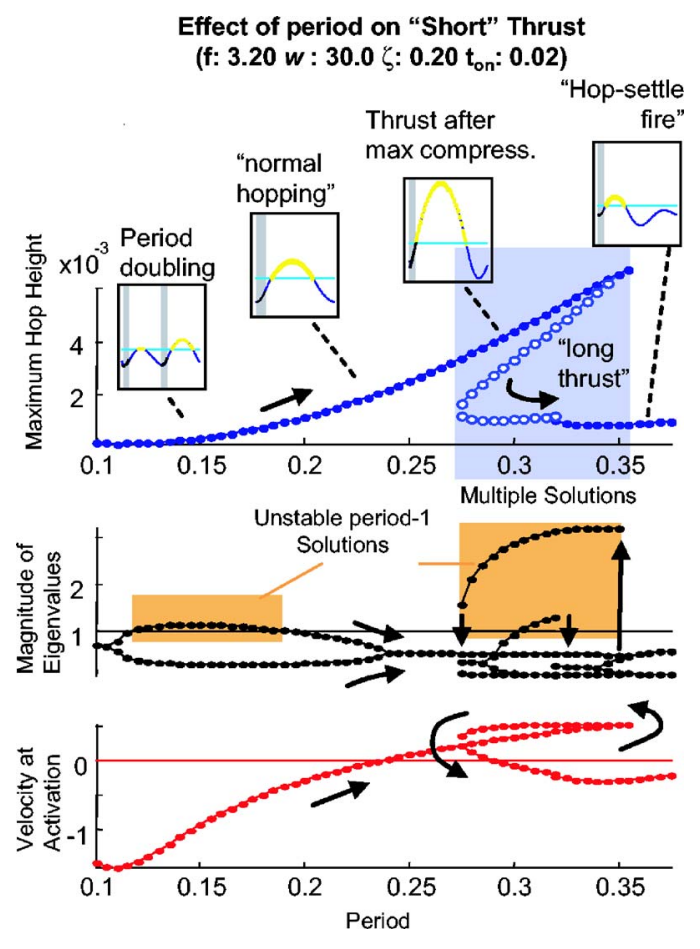

Fig. 6 Steady-state solutions as a function of stride period for the "Short Thrust" case

$250 \mathrm{~ms}$ as the continuum of solutions folds back with respect to stride period. Of the two new sets of solutions available in this range of stride periods, one of them involves activating thrust after maximum compression and is unstable. The instability of these solutions may explain why they were never reported by previous studies [16,17], which found solutions using simulation. The solutions in the second set are termed "Hop-settle-fire" as the mass has started to settle before thrust is applied. These solutions are a result of including both significant damping and the effects of gravity. Previous studies which neglected both gravity and damping did not report these solutions. The hopping heights for these solutions are much lower, but their corresponding eigenvalues are also much lower, and the simulations converge to these solutions.

For the Short Thrust case, Fig. 6 illustrates the effects of changing the open-loop stride period, given a fixed thrust duration $t_{\mathrm{on}}$ $=0.02 \mathrm{~s}$. For short periods, the solutions start out as "Regular Hopping." However, as the period is increased, the eigenvalues start to move outside the unit circle. Simulations for this range are "period-1 unstable" (the state does not repeat after one cycle), but tend to be "period-2 stable" (the state repeats after two cycles). As the period is further increased, the Regular Hopping solutions become period-1 stable again. The velocity at thrust activation also increases, and changes from negative (thrusting before maximum compression) to positive (thrusting after maximum compression). Maximum hopping height is also increased with period and keeps increasing until the continuum of Short Thrust solutions end. At this point, thrust deactivation starts to occur after takeoff, making the solutions Long Thrust. As discussed previously, these Long Thrust solutions in which thrust is activated after maximum compression are unstable, and "fold back" with respect to period. Near 275 ms period another continuum of Hop-settle-fire solutions appears, for which hopping height is lower.

4.2 Maximizing Hopping Height. As shown, hopping height can vary greatly with the stride period. The analysis in Ref. [24] showed that this relationship can be characterized by the net amount of work done by the force function within one loop.

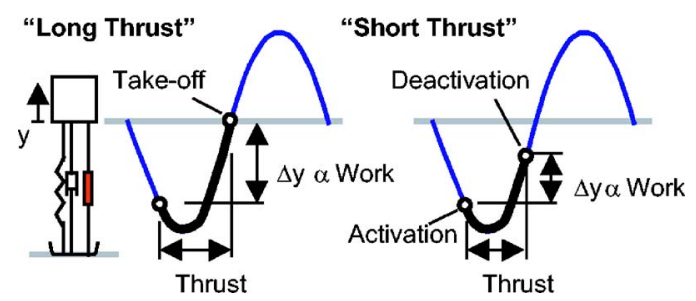

Fig. 7 Work performed by the actuator is proportional to the height difference between the state at thrust activation and thrust deactivation

Steady-state orbits in which the forcing function performs more work will entail higher hopping heights, since the energy that is necessarily dissipated through damping is proportional to the square of the landing velocity (as observed by Ref. [16]), and hopping height is proportional to the square root of the landing velocity. Moreover, the amount of work performed is proportional to the difference in height between thrust activation and deactivation, since the forcing function is constant (see Fig. 7).

The main result of this observation is that for maximal hopping height, it is not always optimal to activate thrust at the leg's maximum compression, as predicated by the Raibert controller, and as found by previous analyses. For short thrust durations, it is more optimal to activate thrust such that deactivation occurs near the point of takeoff.

4.3 Stability for Long Thrust Case. As noted, unstable solutions for the Long Thrust case initiate thrust shortly after maximum compression. These solutions share the same range of stride periods as a set of solutions in which thrust is initiated before maximum compression and a set of Hop-settle-fire solutions (see Fig. 5). In order to visualize what is happening for one such period in this range of multiple solutions, Fig. 8 shows a vector field in the physical state space of solutions for the Poincare Map based at the moment of thrust activation. The spiral curve represents the solutions presented earlier, with the hopping height in the horizontal axis and the velocity on the vertical axis of Fig. 8. The circles are the three simultaneous solutions for a period of $0.26 \mathrm{~s}$. To form the vector field, the model was simulated starting at each point in a grid in the vector space, at the instant of thrust activation. After one period of $0.26 \mathrm{~s}$, a vector line was drawn between

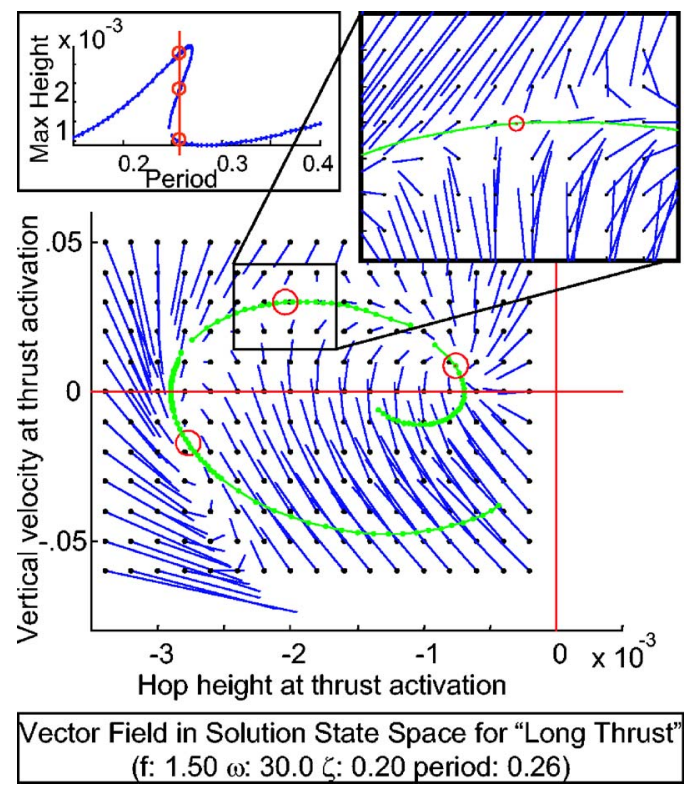

Fig. 8 Unstable steady-state solution for "Long Thrust" case 
the initial point and the state at the end of the simulation, here scaled by 0.4 . Thus, the vector field represents the "flow" of the state sampled discretely at the instant of thrust initiation. Figure 8 helps explain several observed behaviors. First, near periods in which the three solutions start to appear, at the edge of the range of multiple solutions, simulations often exhibited unpredictable or nonperiodic motion. From Fig. 8, one can see how simulations may oscillate nonperiodically between the domains of attraction of the two stable solutions. Second, the domain of attraction of the Hop-settle-fire solution (right-most circle in the vector field) appears to be larger than the domain of the solution in which thrust is initiated before maximum compression (left-most circle). Although both solutions are stable, simulations with periods well into the range of multiple solutions tend to converge to Hop-settle-fire.

From Fig. 8, we can also see that the unstable solutions in which thrust is initiated shortly after maximum compression are "saddle-points," that is, they appear to have one stable mode and one unstable mode. Looking at the Jacobian of the Poincare Map, we can show that these solutions are necessarily unstable.

Starting with the Jacobian as computed using Eq. (48), we first remove the first row and column, since they are zeroes due to the fact that the Poincare Map is based on an event condition which only involves the proxy time variable (in this case, $t=0$ ). We can analyze the stability of the resulting $2 \times 2$ matrix by using the Jury Stability Test [25], which states that a discrete system of two dimensions with the characteristic equation $P(z)$ of the form

$$
P(z)=a_{0} z^{2}+a_{1} z+a_{z}
$$

where $a_{0}>0$ is stable if the following conditions are all satisfied:

$$
\begin{aligned}
\left|a_{2}\right| & <a_{0} a_{0}+a_{1}+a_{2}>0 a_{0}-a_{1}+a_{2}>0\left|\left(a_{0}+a_{2}\right) \cdot\left(a_{2}-a_{0}\right)\right| \\
& >\left|a_{1} \cdot\left(a_{0}-a_{1}\right)\right|
\end{aligned}
$$

Of these conditions, the one that plays a significant role is the third condition. Substituting the elements of the Jacobian found from Eq. (48) into the third condition, expanding all the terms, and simplifying using MATLAB's symbolic toolbox (The Mathworks, Inc.), the third condition becomes

$$
\begin{aligned}
e^{-\zeta w \Delta t_{\mathrm{on}}} & \left(\frac{f}{w_{d} t_{d}}\right) \sin \left(w_{d} \Delta t_{\text {on }}\right)-e^{-\zeta w\left(\tau-2 \Delta t_{a}\right)} \sin \left(w_{d} \Delta t_{\mathrm{off}}\right. \\
- & -2 w_{d} e^{\left.-\zeta w \Delta t_{\mathrm{on}} \dot{y}_{N}\right)>0}
\end{aligned}
$$

where $w_{d}$ is the damped natural frequency of the mass-springdamper system, and $\dot{y}_{N}$ is the velocity at thrust activation. Since the first two products of the expression are always positive, the condition reduces to

$$
\sin \left(w_{d} \Delta t_{\mathrm{on}}\right)-e^{-\zeta w_{n}\left(\tau-2 \Delta t_{a}\right)} \sin \left(w_{d} \Delta t_{\mathrm{off}}\right)-2 w_{d} e^{-\zeta w_{n} \Delta t_{\mathrm{on}} \dot{y}_{N}}>0
$$

From this expression, we can see analytically that stability depends largely on the sign of $\dot{y}_{N}$. For solutions in which thrust occurs after maximum compression, $\dot{y}_{N}$ is positive, which makes the third term in the expression negative. Since $\Delta t_{\text {on }}$ is approximately equal to $\Delta t_{\text {off }}$ for these solutions, this third term, and thus the sign of $\dot{y}_{N}$, plays a large role in determining stability, regardless of thrust magnitude or other system parameters.

Intuitively, this can be explained by looking at the effect on work input that perturbations about steady state have for solutions in which thrust is activated after maximum compression. As illustrated in Fig. 9, thrusting "late," when it occurs after maximum compression, causes a decrease in work, a subsequent shorter airborne phase, and an even "later" thrust initiation in the next cycle, resulting in unstable behavior.

4.4 Stability for Short Thrust Case. In the Short Thrust case, solutions in which thrust is initiated after maximum compression are not necessarily unstable as in the Long Thrust case.

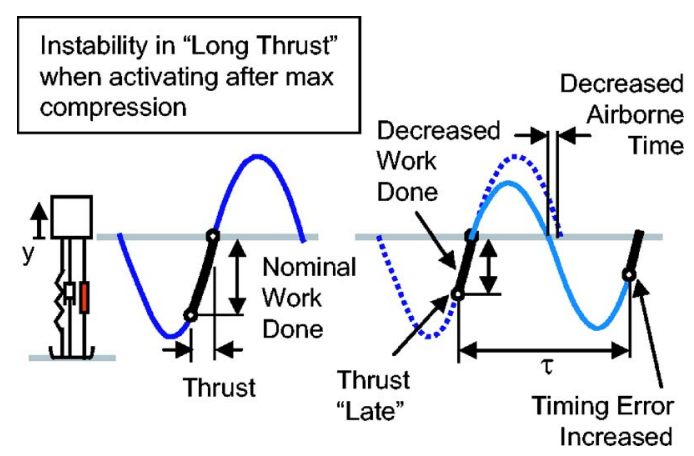

Fig. 9 Effect of perturbations in the timing of thrust activation on the work performed, and resulting takeoff velocity and airborne phase duration for the "Long Thrust" case

In this case, initiating thrust late moves both thrust activation and deactivation later, which increases the work done, causing a longer airborne phase and a correction to the error in thrust timing.

As shown previously in Fig. 6, instability for the Short Thrust case occurs in two ranges of periods. The first is in short periods for which thrust activation occurs well before maximum compression and hopping height is low. The second is when period is increased such that thrust activation occurs after maximum compression and thrust deactivation occurs after takeoff, in which case the system becomes Long Thrust and is unstable as explained in the previous section. The first case is of interest, and is visualized in Fig. 10. This figure shows a vector field similar to the one presented in Fig. 8, but for the Short Thrust solutions, and for a period in which the eigenvalues are unstable. Here the vectors are directed toward the steady-state solution, but with eigenvalues that have a magnitude greater than one.

This is confirmed by looking at the expression for the Jacobian of the Short Thrust case, found from Eq. (49). In this case, we can remove the second row and second column, since the Poincare Map is based on the moment of landing $(y=0)$. This Jacobian is, after simplification, equal to

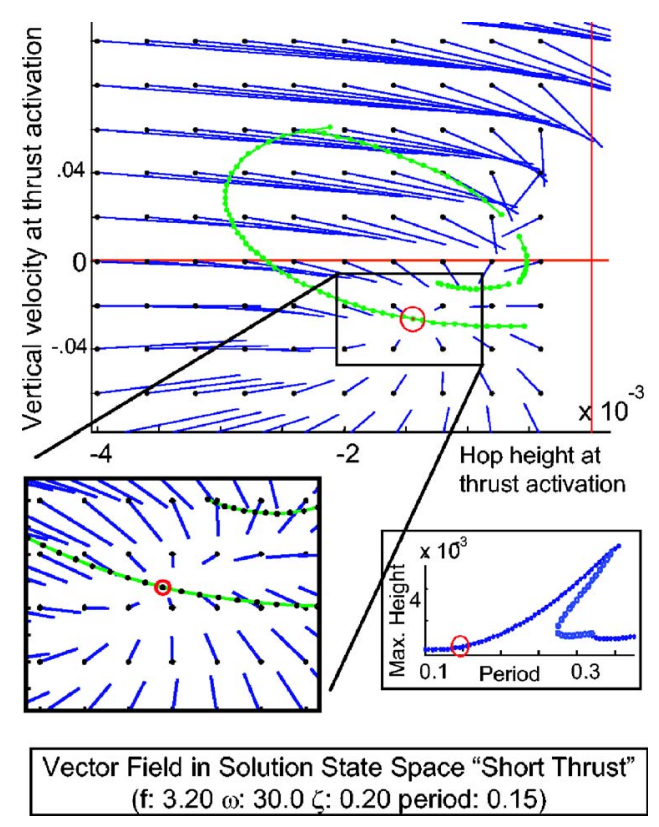

Fig. 10 Unstable steady-state solution for "Short Thrust" case for a period of 0.15 . At this period, thrust is initiated well before maximum compression, and takeoff velocity is small. 


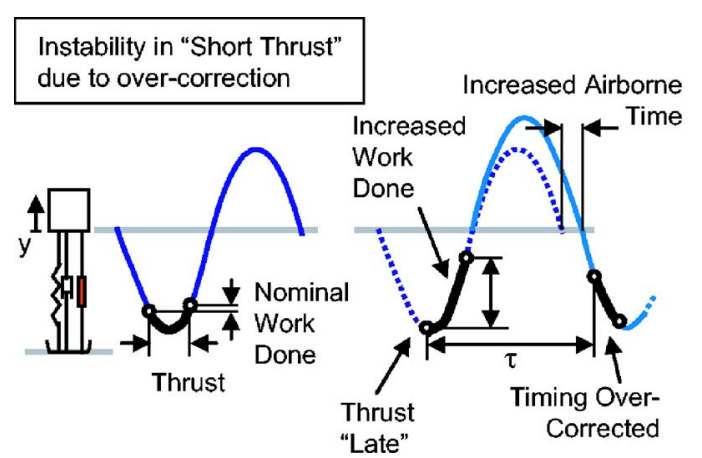

Fig. 11 Effect of perturbations in the timing of thrust activation on the work performed, and resulting takeoff velocity and airborne phase duration for the "Short Thrust" case

$$
\begin{aligned}
\tilde{J}_{S T}= & -\frac{1}{\dot{y}_{T}}\left[\begin{array}{cc}
1 & 2 \\
0 & -1
\end{array}\right] \\
& \times\left[\begin{array}{cc}
1 & 0 \\
\left(-1-2 \zeta w \dot{y}_{T}\right) & -\dot{y}_{T}
\end{array}\right] e_{\text {stance }}^{A \Delta t}\left[\begin{array}{cc}
\dot{y}_{T} & 0 \\
\left(1-2 \zeta w \dot{y}_{T}\right) & 1
\end{array}\right]
\end{aligned}
$$

where $\widetilde{J}_{S T}$ is the reduced Jacobian, $\dot{y}_{T}$ is the vertical velocity at takeoff, and $\Delta t_{\text {stance }}$ the total stance time.

From this expression, we can see what can happen for solutions in which thrust is activated well before maximum compression. For these solutions, $\dot{y}_{T}$ is small (but positive, since it is the velocity at takeoff) and the stance time, $\Delta t_{\text {stance }}$, is approximately (but less than) one half of the mass-spring-damper system's damped natural period, $\tau_{d}$. Using this approximation

$$
\Delta t_{\text {stance }} \approx \tau_{d} / 2
$$

$e^{A \Delta t_{\text {stance }}}$ can be approximated as follows

$$
e_{\text {stance }}^{A \Delta t} \approx e^{-\zeta w_{n} \Delta t_{\text {stance }}}\left[\begin{array}{cc}
-1 & 0 \\
-b & -1
\end{array}\right]
$$

where $b$ is a number equal to

$$
b=w_{n} \sin \left(w_{d} \Delta t_{\text {stance }}\right) / \sqrt{1-\zeta^{2}}
$$

The expression for the Jacobian then becomes

$$
\tilde{J}_{S T} \approx-e^{-\zeta w_{n} \Delta t_{\text {stance }}}\left[\begin{array}{cc}
3+2 \dot{y}_{T} b & 2 \\
-2-\dot{y}_{T} b & -1
\end{array}\right]
$$

where

$$
a=2 \zeta w \dot{y}_{T}
$$

The eigenvalues of this Jacobian are

$$
-e^{-\zeta w_{n} \Delta t_{\text {stance }}}\left(\left(1+\dot{y}_{T} b\right) \pm \sqrt{\left(1+\dot{y}_{T} b\right)^{2}-1}\right)
$$

From this simple expression of the eigenvalues, we can see that both eigenvalues will be real and negative if $\dot{y}_{T} b$ is small $\dot{y}_{T} b$ is approximately 0.2 for the case shown in Fig. 10). If $\dot{y}_{T}$ and $\Delta t_{\text {stance }}$ are such that the larger eigenvalue is unstable, it will be because it is less than -1 . We also observe from this result that increasing the damping ratio has the effect of stabilizing this eigenvalue. A negative eigenvalue less than -1 implies that the system "overcompensates" or "overcorrects" for disturbances. This is illustrated in Fig. 11. In this situation, thrusting late will move both thrust activation and deactivation further along the cycle, decreasing the amount of negative work performed and increasing the amount of positive work. This large increase in the net work can cause a very large increase in the takeoff velocity and ensuing airborne phase such that thrust occurs "very early" from the nominal timing, overcompensating for the initial disturbance. This nature of the instability in this range of periods helps explain why

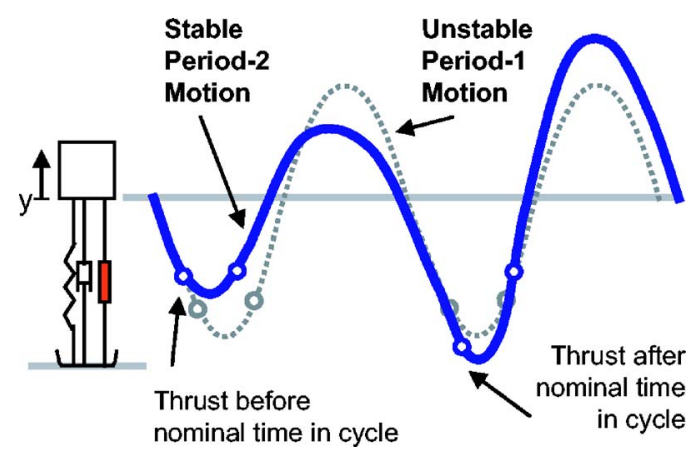

Fig. 12 Stride periods for which unstable period-1 trajectories exist in the Short Thrust case tend to result in period-2 stable trajectories

simulations in this range tend to be period-2 trajectories. These period-2 trajectories repeat themselves after two stride cycles, and tend to "oscillate" about the unstable period-1 trajectory, as illustrated in Fig. 12. The negative sign of the eigenvalue indicates that perturbations in the time that thrust is activated along the period-1 trajectory will "overshoot" at the next cycle, causing thrust activation to occur alternately before and after the nominal point in the period-1 cycle.

\section{Experimental Validation}

In order to verify that these phenomena occur in physical systems, this section presents experimental data for a vertical hopping machine called the "Dashpod." The Dashpod is illustrated in Fig. 13, and consists of a low-stiction pneumatic piston attached to a wide curved dish "foot" on which it stands with a spring in parallel. The machine hops by activating the solenoid valve with a square wave. The curved foot stabilizes the machine when in contact with the ground. More details on the machine can be found in Ref. [26].

Figure 14 shows experimental data of the Dashpod while hopping. The data were taken through hi-speed video capture of reflective markers placed on the machine's body and foot. Figure 14 shows nine experimental trials, in which the mass of the body and the stride period were varied. For each trial, the plot shows the time history of the height of the body and foot for several consecutive stride periods.

In Fig. 14, the left-most column of trials represents hopping trajectories with short stride period compared to the dynamics of the system. As shown, thrust activation occurs before maximum compression, and thrust deactivation occurs shortly after. More interestingly, close examination of the hopping trajectories shows that the motion is period 2. This is most evident in the top-most trial, in which no mass was added, and more subtle in the other trials. This validates the observations made for the Short Thrust case in simulation and analytically in which period-2 behavior appears as a possible consequence of the overcorrecting effect.
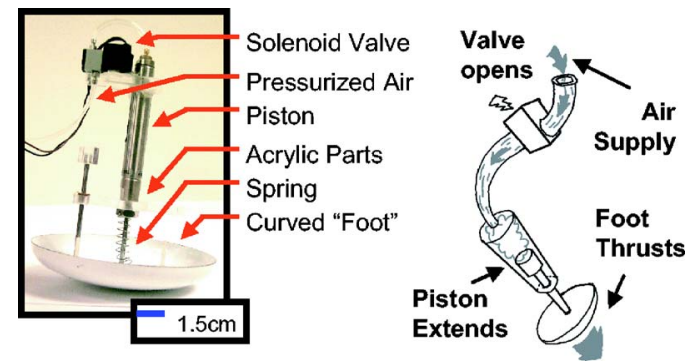

Fig. 13 The "Dashpod," a pneumatically actuated vertical hopper 


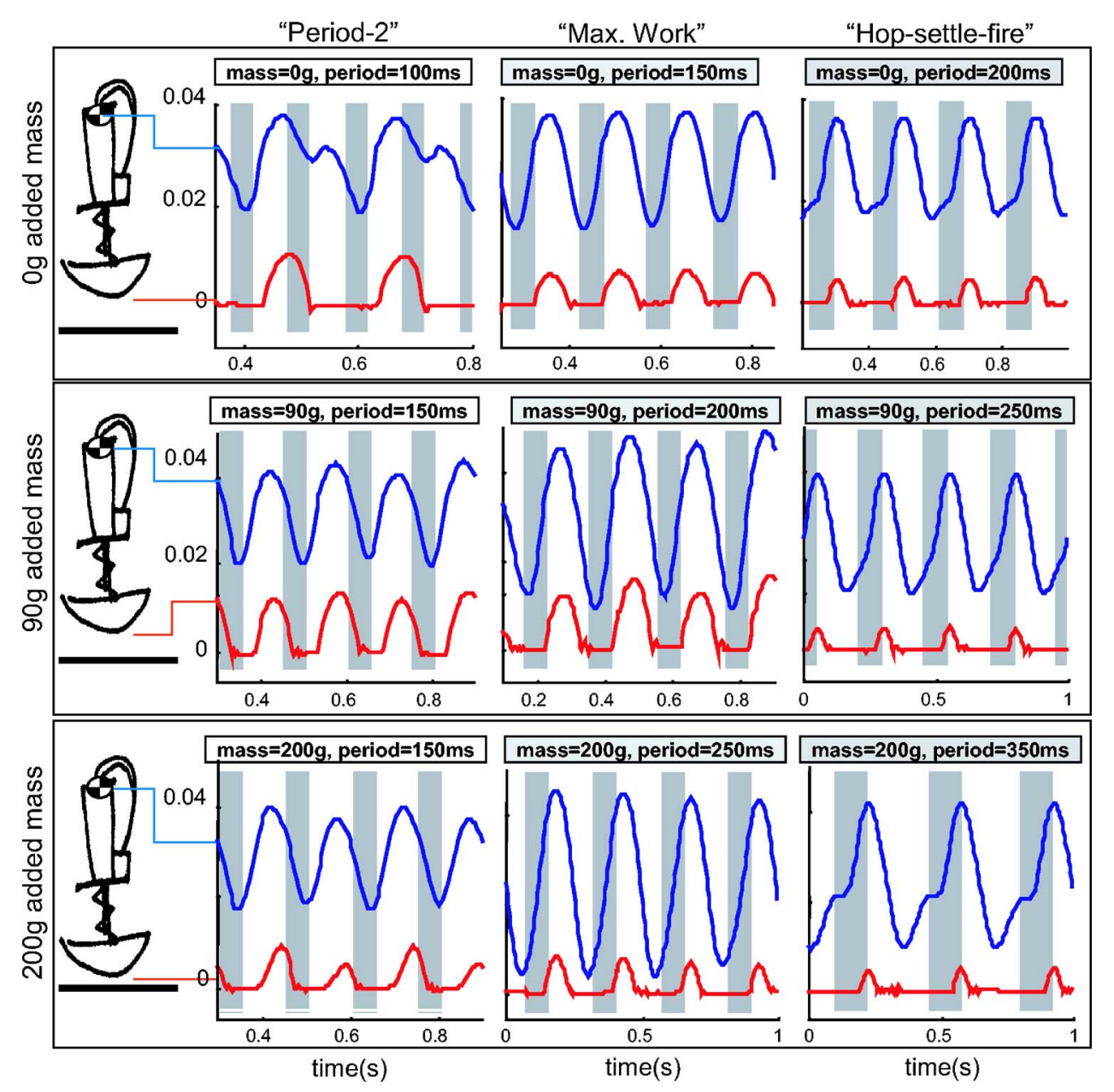

Fig. 14 Experimental data of hopping trajectories for the Dashpod vertical hopper

The center column of trials represents hopping trajectories in which the hopper is activated with a period that is closer to the natural period of the hopper. As shown, thrust activation occurs near maximum compression of the spring, and deactivation occurs near lift-off, maximizing the work performed by the actuator, as evident in the high oscillations of the hopper body. Also of interest is the indication that these trajectories may be on the border of instability. As shown, the body trajectories for the trials with added mass show nonperiodic irregularities in maximum hop height. Although this non-period-1 motion may be due to unmodeled dynamics, the analysis in this paper suggests that this behavior may be due to the unstable effect when activating thrust near maximum compression.

Finally, the right-most column of experimental trials shows Hop-settle-fire hopping trajectories. These trajectories are due to gravity and damping when the stride period is much higher than the natural period and have smaller hopping heights, as the model predicted.

\section{Conclusions and Future Work}

All of these observations point to insights about the fundamental behavior of dynamic open-loop hopping systems. Foremost is the apparent trade-off between performance and stability in openloop vertical hopping. As shown, hopping trajectories with maximum hopping height were found to be unstable or near regions of instability. Moreover, it was found that multiple steady-state trajectories can exist for a given period of the motor pattern, a result not previously reported. This indicates a loss of control, as the system may converge to any one of the solutions available. Re- sults indicate that these multiple solutions exist near solutions of optimal hopping height, and that the system will often converge to suboptimal, but more stable, trajectories. As a result, a fundamental limitation of open-loop hopping system is that they may need to be operated at suboptimal stride periods to avoid unstable or undesirable behavior.

This trade-off was characterized in the analysis by looking at the dynamics from the perspective of the net amount of work performed by the actuator and how disturbances affect it between cycles of the trajectory. Depending on the relative phasing of the activation pattern to the motion of the system in a given steadystate trajectory, the actuator may perform positive and negative work, and maximum hopping height corresponds to trajectories in which maximum work is done. Unstable steady-state trajectories can be characterized by the changes in the work performed by perturbations in the timing of thrust activation and deactivation. For the Long Thrust case, analytical and numerical results show that trajectories in which thrust is initiated shortly after maximum compression are unstable, as changes in the thrust timing result in unfavorable changes in the work performed that increase the timing perturbation in the subsequent stride cycle. This can lead to uncontrolled and unstable hopping, as has been observed in both simulation and physical robotic hoppers. For the Short Thrust case, analytical and numerical results show that trajectories with short periods (or high frequencies) can be unstable with an eigenvalue less than -1 . This large negative eigenvalue indicates that perturbations in the thrust timing cause changes in the work performed that overcompensate for the perturbation in the next stride 
cycle. This helps explain the circumstances under which period-2 behavior has been observed in simulation and the physical hopper.

These insights are postulated to be applicable to hopping systems in general, and, as shown in Ref. [24], play a large role in helping characterize the overall behavior of the Sprawl hexapedal robots. Observations about the relationship between work performed and the timing of thrust activation have led to the development of adaptation strategies for online optimization of the frequency of the robot's motor pattern [24]. Furthermore, similar conclusions have been found for a planar SLIP-like running model $[27,28]$.

The framework utilized and the analytical formulas derived in this paper can be applied to the study of periodic orbits in other linear time invariant hybrid systems. The general formulation presented will allow future work to explore the stability properties of control strategies with combined open-loop (time-based) and closed-loop (sensor-based) thrust activation, which studies suggest animals may employ for robustness and versatility [2,29].

\section{Acknowledgment}

We have been fortunate to collaborate with Professor Robert J. Full at the U.C. Berkeley Polypedal Laboratory. Thanks especially to Jonathan Karpick and the other members of the Biomimetics lab at CDR. Funding was provided by the Office of Naval Research under Grant No. N00014-98-1-0669.

\section{References}

[1] Rossignol, S., Lund, J. P., and Drew, T., "The Role of Sensory Inputs in Regulating Patterns of Rhythmical Movements in Higher Vertebrates," 1998, in Neural Control of Rhythmic Movements in Vertebrates, A. H. Cohen, S. Rossignol, and S. Grilner, eds., John Wiley \& Sons, Hoboken, NJ.

[2] MacKay-Lyons, M., 2002, "Central Pattern Generation of Locomotion: A Review of the Evidence," Phys. Ther., 82(1), pp. 69-83.

[3] Dietz, V., Schmidtbleicher, D., and Noth, J., 1979, "Neuronal Mechanisms of Human Locomotion," J. Neurophysiol., 42(5), pp. 1212-1222.

[4] Orlosvky, G. N., Deliagnia, T. G., and Grilner, S., 1999, Neuronal Control of Locomotion, Oxford University Press, New York

[5] Cham, J. G., Bailey, S. A., Clark, J. E., Full, R. J., and Cutkosky, M. R., 2002, "Fast and Robust: Hexapedal Robots via Shape Deposition Manufacturing," Int. J. Robot. Res., 21(10), pp. 869-883.

[6] Saranli, U., Buehler, M., and Koditschek, D. E., 2001, "RHex: A Simple and Highly Mobile Hexapod Robot,” Int. J. Robot. Res., 20, pp. 616-631.

[7] McGeer, T., 1990, "Passive Dynamic Walking," Int. J. Robot. Res., 9(2), pp. 62-82.

[8] McMahon, T. A., 1999, Muscles, Reflexes and Locomotion, University Press, Princeton, NJ.

[9] Brown, I. E., and Loeb, G. E., 1999, “A Reductionist Approach to Creating and
Using Neuromusculoskeletal Models," in Biomechanics and Neural Control of Posture and Movement, Springer, New York.

[10] Kubow, T. M., and Full, R. J., 1999, "The Role of the Mechanical System in Control: A Hypothesis of Self-Stabilization in Hexapedal Runners." Philos. Trans. R. Soc. London, Ser. B, 354, pp. 849-862.

[11] Cavagna, G. A., Heglund, N. C., and Taylor, C. R., 1975, "Walking, Running, and Galloping: Mechanical Similarities Between Different Animals," Scale Effects in Animal Locomotion, Proceedings of an International Symposium, T. J. Pedley, Academic, New York, pp. 111-125.

[12] Raibert, M. H., 1986, Legged Robots that Balance, MIT, Cambridge. MA.

[13] DeCarlo, R., Branicky, M., Pettersson, S., and Lennartson, B., 2000, "Perspectives and Results on the Stability and Stabilizability of Hybrid Systems," Proc. IEEE, 88(7), pp. 1069-1082.

[14] Koditschek, D. E., and Buehler, M., 1991, "Analysis of a Simplified Hopping Robot," Int. J. Robot. Res., 10(6), 587-605.

[15] Vakakis, A. F., Burdick, J. W., and Caughey, T. K., 1991,"An Interesting Strange Attractor in the Dynamics of a Hopping Robot,” Int. J. Robot. Res. 10, 606-618.

[16] Ringrose, R., 1997, "Self-Stabilizing Running," Ph.D. thesis, MIT, Cambridge, MA.

[17] Berkemeier, M. D., and Desai, K. V., 1998, "A Comparison of Three Approaches for the Control of Hopping Height in Legged Robots," International Journal of Robotics Research (submitted).

[18] Komsuoglu, H., and Koditschek, D. E., 2000, "Preliminary Analysis of a Biologically Inspired 1-DOF 'Clock' Stabilized Hopper," Proceedings of World Multiconference on Systemics, Cybernetics and Informatics (SCI2000), Orlando, July 23-26, Vol. IX, pp. 670-675.

[19] Meijer, K., and Full, R. J., 2007, "Stabilizing Properties of Invertebrate Skeletal Muscle," American Zoologist (in press)

[20] Full, R. J., Autumn, K., Chung, J. I., and Ahn, A., 1998, "Rapid Negotiation of Rough Terrain by the Death-Head Cockroach," Am. Zool., 38, p. 81A.

[21] Garcia, M., Kuo, A., Peattie, A. M., Wang, P. C., and Full, R. J., 2000, "Damping and Size: Insights and Biological Inspiration," in Proceedings of the International Symposium on Adaptive Motion of Animals and Machines, Montreal, Canada.

[22] Sastry, S., 1999, Nonlinear Systems: Analysis, Stability and Control, Springer, Berlin.

[23] Mombaur, K. D., 2001, "Stability Optimization of Open-Loop Controlled Walking Robots," Ph.D. thesis, Universitat Heidelberg, Heidelberg.

[24] Cham, J. G, Karpick, J., and Cutkosky, M. R., 2004, "Stride Period Adaptation for a Biomimetic Running Hexapod," Int. J. Robot. Res., 23(2), pp. 141-154.

[25] Ogata, K., 1994, Discrete-Time Control Systems, Prentice-Hall, Englewood Cliffs, NJ.

[26] Cham, J. G., Stafford, B., and Cutkosky, M. R., 2001, "See Labs Run: A Design-riented Laboratory for Teaching Dynamic Systems," ASME, IMECE 2001, Nov. 11-16, New York.

[27] Cham, J. G., 2002, "On Performance and Stability in Open-Loop Hopping," $\mathrm{Ph} . \mathrm{D}$. thesis, Stanford University, Stanford.

[28] Cham, J. G., and Cutkosky, M. R., 2003, “Adapting Work Through Actuator Phasing in Running," 2003 International Symposium Adaptive Motion of Animals and Machines (AMAM2003), Kyoto, Japan.

[29] Abbas, J., and Full, R. J., 2000, "Neuro-Mechanical Interaction in Cyclic Movements," Biomechanics and Neural Control of Posture and Movement, Springer, Berlin, pp. 177-191. 